\title{
Optical line emission from the supernova remnant G 73.9+0.9
}

\author{
F. Mavromatakis ${ }^{\star}$ \\ University of Crete, Physics Department, PO Box 2208, 71003 Heraklion, Crete, Greece \\ Received 12 August 2002 / Accepted 25 October 2002

\begin{abstract}
Flux calibrated images of the field around the known supernova remnant G 73.9+0.9 in the $\mathrm{H} \alpha+\left[\mathrm{N}_{\text {II }}\right]$, [S II], [O II] and [O III] emission lines are presented. The low ionization images are characterized by diffuse emission both within and outside the extent of the remnant. The flux calibrated images revealed a few small scale structures in the east areas of G 73.9+0.9. The long-slit spectra identify the emission from one of them as emission from shock heated gas. This patchy structure is $\sim 3^{\prime}$ long and emits $\mathrm{H} \alpha$ flux at a level of $\sim 33 \times 10^{-17} \mathrm{erg} \mathrm{s}^{-1} \mathrm{~cm}^{-2} \operatorname{arcsec}^{-2}$. The bright diffuse arc-like structure in the center of the field seems to be associated to $\mathrm{G} 73.9+0.9$ given its spectral signature and positional relation with the non-thermal radio emission. A $\sim 8^{\prime}$ long filamentary structure with an absolute $\mathrm{H} \alpha$ flux of $\sim 9 \times 10^{-17} \mathrm{erg} \mathrm{s}^{-1} \mathrm{~cm}^{-2} \operatorname{arcsec}^{-2}$ is detected in the [O III] emission line to the south of $\mathrm{G} 73.9+0.9$ but is probably unrelated. The deep long-slit spectra suggest complete recombination zones, shock velocities below $90 \mathrm{~km} \mathrm{~s}^{-1}$, low electron densities $\left(<50 \mathrm{~cm}^{-3}\right)$ and non-negligible magnetic field strengths.
\end{abstract}

Key words. ISM: general - ISM: supernova remnants - ISM: individual objects: G 73.9+0.9

\section{Introduction}

Initial detection of the galactic remnant G $73.9+0.9$ was reported by Reich et al. (1986) who observed non-thermal radio emission over an extent of $\sim 30^{\prime} \times 30^{\prime}$. Wider field, higher resolution observations at $1420 \mathrm{MHz}$ revealed emission extending for more than $\sim 1^{\circ}$ towards larger galactic longitudes (Pineault $\&$ Chastenay 1990). However, this extended emission in the north is not related to the actual supernova remnant emission in the south since Pineault (1998) has shown that it originates from a bipolar $\mathrm{H}$ II region. Optical interferometric observations in $\mathrm{H} \alpha$ have been performed by Lozinskaya et al. (1993) at several locations within the remnant's extent and its neighbourhood. It was found that the width of the $\mathrm{H} \alpha$ line was broader over the remnant than in the surrounding medium and the nebula's expansion velocity was proposed to lie below $\sim 50 \mathrm{~km} \mathrm{~s}^{-1}$. A kinematic distance of $0.5-2.0 \mathrm{kpc}$ is consistent with the radial velocity measurements of Lozinskaya et al. (1993). The HI images obtained by Pineault et al. (1996) at several velocity intervals do not show unambiguous evidence of gas associated with the remnant. Gas approaching us with a local standard of rest (LSR) velocity of $-45 \mathrm{~km} \mathrm{~s}^{-1}$ shows a morphology similar to that of the radio continuum emission but it is not clear whether it is related or not to the remnant Pineault et al. (1996). The Wolf-Rayet stars WR 134, 135 and 137 are located in the immediate vicinity of the remnant but none of them has been shown to interact with it (Pineault et al. 1996; Gervais \& St-Louis 1999).

\footnotetext{
* e-mail: fotis@physics.uoc.gr
}

Flux calibrated CCD images of moderate resolution in major optical emission lines were obtained in order to identify the remnant's optical emission and study the interaction of the remnant with the ISM. In addition, deep long-slit spectroscopy at selected areas of interest was performed. Information about the observations and the data reduction is given in Sect. 2. In Sects. 3 and 4 the results of the imaging and spectral observations are presented. Finally, in Sect. 5 the properties of the remnant and its environment are discussed.

\section{Observations}

\subsection{Optical images}

The current wide field observations were performed with the $0.3 \mathrm{~m}$ Schmidt-Cassegrain telescope at Skinakas Observatory, Crete, Greece. The field around G 73.9+0.9 was observed on July 12 and 13, 1999. The imaging observations were performed with a $1024 \times 1024$ SITe CCD which offers a $89^{\prime} \times 89^{\prime}$ field of view and an image scale of $5^{\prime \prime}$ per pixel. The remnant was observed through the $\mathrm{H} \alpha+\left[\mathrm{NII}_{\mathrm{II}}\right.$, [S $\left.\mathrm{S}_{\mathrm{II}}\right]$, [O II], and [O III] filters for $1800 \mathrm{~s}$ in each one with this highly efficient CCD chip. Details on the filter characteristics can be found in Mavromatakis et al. (2002). The astrometric solutions of the source data made use of the HST Guide star catalogue (Lasker et al. 1999) and all coordinates quoted in this work refer to epoch 2000. All data frames have been projected to a common origin on the sky.

Standard IRAF and MIDAS routines were employed for the reduction of the data. All frames were bias subtracted and flat-field corrected using a series of well exposed twilight 


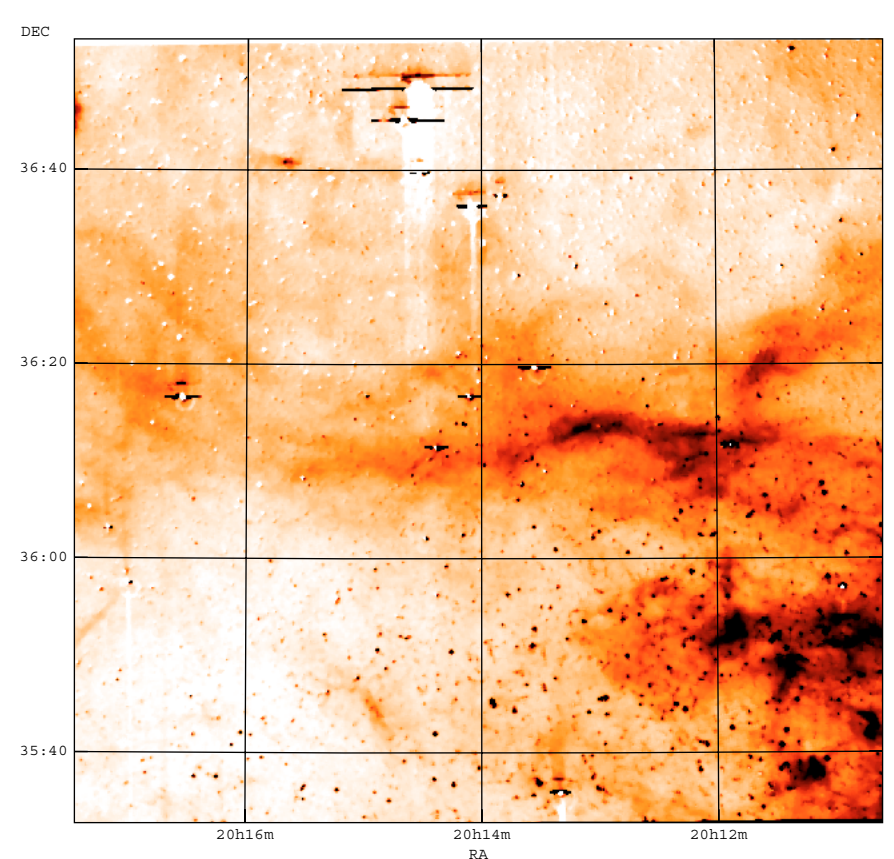

Fig. 1. The major characteristic of the $\mathrm{H} \alpha+[\mathrm{N}$ II $]$ line image is the diffuse and extended optical emission in the field of $\mathrm{G} 73.9+0.9$. The image has been smoothed to suppress the residuals from the imperfect continuum subtraction. The shadings run linearly from 0 to $250 \times 10^{-17} \mathrm{erg} \mathrm{s}^{-1} \mathrm{~cm}^{-2} \operatorname{arcsec}^{-2}$. The line segments seen near overexposed stars in this figure and the next figures are due to the blooming effect. The declination axis unit, in this figure and the next figures, is given in the format dd:mm and all coordinates are given for epoch 2000 .

flat-fields. The absolute flux calibration was performed through observations of a series of spectrophotometric standard stars (HR 5501, HR 7596, HR 7950 and HR 8634; Hamuy et al. 1992, 1994).

\subsection{Optical spectra}

Long-slit spectra were aquired on July 4, and 5, 2002 with the $1.3 \mathrm{~m}$ Ritchey-Cretien telescope at Skinakas Observatory. The data were taken with a 1300 line $\mathrm{mm}^{-1}$ grating and a $800 \times 2000$ SITe CCD covering the range of $4750 \AA-6815 \AA$. The slit had a width of 7'. 7 and a length of 7'.9 and, in all cases, was oriented in the south-north direction. The coordinates of the slit centers, the number of available spectra from each location and the total exposure times are given in Table 1. The spectrophotometric standard stars HR 5501, HR 7596, HR 7950, and HR 8634 were observed for the absolute calibration of the available spectra.

\section{The imaging observations}

\subsection{The $H \alpha+[N 川]$ and $\left[S_{I} I\right]$ line images}

The images of the area covering G 73.9+0.9 show the known arc of diffuse emission (e.g. Fig. 4 of Pineault et al. 1996) as well as part of LBN $074.83+00.62$ in the north-east edge of our field and NGC 6883 and LBN 073.10+01.41 in the south-west. The morphology seen in the $\mathrm{H} \alpha+\left[\mathrm{N}_{\text {II }}\right]$ image

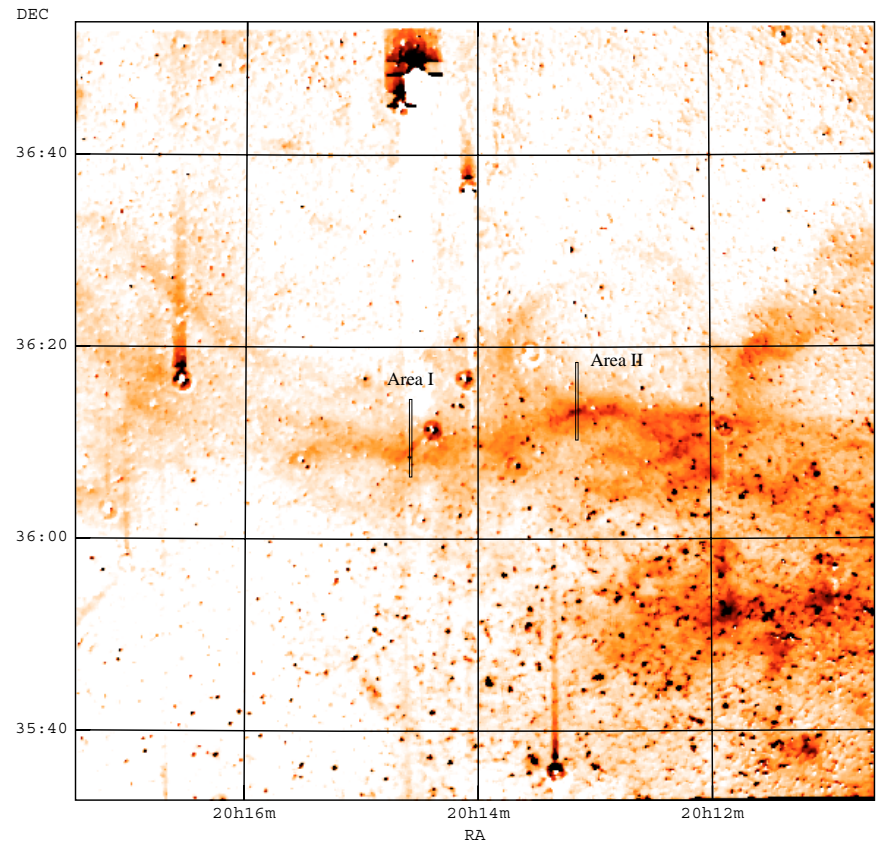

Fig. 2. The field of G 73.9+0.9 imaged with a filter that isolates the sulfur lines at 6716 and $6731 \AA$. The image has been smoothed to suppress the residuals from the imperfect continuum subtraction, while the shadings run linearly from 0 to $50 \times 10^{-17} \mathrm{erg} \mathrm{s}^{-1} \mathrm{~cm}^{-2} \operatorname{arcsec}^{-2}$. The long rectangles show the approximate projections of the slits on the sky. Two lanes of diffuse emission extend to the north-east of area I.

Table 1. Spectral log.

\begin{tabular}{lll}
\hline \hline Slit centers & & Exp. times $^{\mathrm{a}}$ \\
\hline$\alpha$ & $\delta$ & (No. of spectra $^{\mathrm{b}}$ ) \\
\hline $20^{\mathrm{h}} 14^{\mathrm{m}} 36^{\mathrm{s}}$ & $36^{\circ} 10^{\prime} 21^{\prime \prime}$ & $7800(2)$ \\
\hline $20^{\mathrm{h}} 13^{\mathrm{m}} 10^{\mathrm{s}} .8$ & $36^{\circ} 15^{\prime} 33^{\prime \prime}$ & $7800(2)$ \\
\hline $20^{\mathrm{h}} 14^{\mathrm{m}} 41^{\mathrm{s}} .9$ & $35^{\circ} 48^{\prime} 25^{\prime \prime}$ & $7800(2)$ \\
\hline
\end{tabular}

a Total exposure times in $\mathrm{s}$.

${ }^{\mathrm{b}}$ Number of spectra obtained.

(Fig. 1) is generally similar to that of the [S II] image (Fig. 2) since both are dominated by diffuse emission. The [S II] emission is not continuous along the arc but gaps in intensity are present. Especially, small sized structures $\left(\sim 1^{\prime}-3^{\prime}\right)$ and weak diffuse emission are present to the east of $20^{\mathrm{h}} 13^{\mathrm{m}} 20^{\mathrm{s}}$. These flux variations may reflect inhomogeneities in the interstellar "clouds" where the optical emission originates. No filamentary structures are detected in these low ionization images. The $\mathrm{H} \alpha+\left[\mathrm{N} \mathrm{II}_{\text {II }}\right.$ and [S II] images being flux calibrated provide a useful tool to identify, at least to a first approximation, the nature of the observed emission. Careful examination of the ratio of the [S $\mathrm{II}]$ to $\mathrm{H} \alpha+[\mathrm{N}$ II] images shows that there is an area which seems to have a ratio higher by $\sim 25 \%$ than that measured in areas of strong diffuse emission. This location (area I in Fig. 2) is found $\sim 3^{\prime}$ to the south-east of the bright star GSC 2683-3728 and extends for $\sim 11^{\prime}$ in the east-west direction. We note here that the ratio map is too noisy to display. Two lanes of diffuse emission are detected $\sim 15^{\prime}$ to the north-east of area I which extend further in the same direction for $\sim 26^{\prime}$. These lanes are also 
present in the POSS plates. Finally, a $\sim 5^{\prime}$ long structure is detected around $\alpha=20^{\mathrm{h}} 14^{\mathrm{m}} 55^{\mathrm{s}}$ and $\delta=35^{\circ} 44^{\prime} 44^{\prime \prime}$. The nature of this structure as well as that of the two lanes is not clear since the flux calibrated images indicate a $\left[\mathrm{S}_{\mathrm{II}}\right] / \mathrm{H} \alpha$ ratio of $\sim 0.4$. Several very bright stars lie within the field of G 73.9+0.9 and have been overexposed.

\subsection{The $[\mathrm{O} I]$ and $[\mathrm{O} I I]$ images}

The [O II] image (Fig. 3) displays basically the same morphological features seen in the $\mathrm{H} \alpha+\left[\mathrm{N}_{\mathrm{II}}\right]$ and $\left[\mathrm{S}_{\mathrm{II}}\right]$ images discussed in Sect. 3.1. A broken loop of faint emission is detected immediately to the north of the arc of diffuse emission and is designated by the dashed line in Fig. 3. This loop is also present in the [S $\mathrm{II}]$ and $\mathrm{H} \alpha+\left[\mathrm{N}_{\mathrm{II}}\right]$ images but appears less well defined (Figs. 1, 2). In Fig. 4 we show the correlation between the low ionization lines and the radio emission at $1420 \mathrm{MHz}$ (Pineault \& Chastenay 1990). In this figure it is also seen that the north part of the loop seems to extend outside the bulk of the $1420 \mathrm{MHz}$ emission. In addition, this structure emits weak sulfur emission relative to $\mathrm{H} \alpha$ and may be an $\mathrm{H}$ in region.

Contrary to other remnants showing well defined [O III] filamentary emission (e.g. Boumis et al. 2002 on G 17.4-2.3), the field of G 73.9+0.9 is dominated by diffuse emission, especially prominent all along the western edge of the field (Fig. 5). The arc of diffuse emission seen in the low ionization images is also present in this medium ionization line but appears shorter and basically extends only up to $\alpha \simeq 20^{\mathrm{h}} 13^{\mathrm{m}} 30^{\mathrm{s}}$. This is in agreement with the absence of [O III] emission in the spectrum obtained from area I (Sect. 4). The [O III] image also revealed the presence of a filamentary structure to the south. It is centered at $\alpha=20^{\mathrm{h}} 14^{\mathrm{m}} 42^{\mathrm{s}}$ and $\delta=34^{\circ} 47^{\prime} 20^{\prime \prime}$ and extends for $\sim 8^{\prime}$ in the south-east to north-west direction (Fig. 5). Since no obvious evidence for emission is seen in the $\mathrm{H} \alpha+\left[\mathrm{N} \mathrm{II}_{\mathrm{II}}\right.$ and [S II] images, deep spectra were obtained in order to study this structure in more detail and the spectral results are discussed in Sect. 4. Radio emission does not seem associated with this filamentary structure.

\section{The long-slit spectra from $G 73.9+0.9$}

The deep low resolution spectra were taken at selected areas based on the images obtained with the $0.3 \mathrm{~m}$ telescope. The optical spectrum obtained from area I (Fig. 2) definetely suggests that the observed emission originates from shock heated gas since a ratio of $[\mathrm{S} \mathrm{II}] / \mathrm{H} \alpha$ of $\sim 0.7$ is measured (Table 2 ). This ratio is greater than 0.4 , typically greater than 0.5 , in known SNRs (e.g. Smith et al. 1993; Raymond et al. 1988), while in $\mathrm{H}$ II regions is found below 0.35 , typically around 0.2 (e.g. Hunter et al. 1992). The $\mathrm{H} \alpha / \mathrm{H} \beta$ ratio indicates moderate interstellar extinction equivalent to a logarithmic extinction $c$ of $0.81 \pm 0.03$ or an $E(B-V)$ of $0.54 \pm 0.02$. The formulas used to derive these values are $c=1 / 0.331 \cdot \log \left((\mathrm{H} \alpha / \mathrm{H} \beta)_{\mathrm{obs}} / 3\right)$ and $E(B-V)=0.664 \cdot c($ Kaler 1976; Aller 1984). This small scale structure is quite strong in terms of flux since the absolute $\mathrm{H} \alpha$ flux is $\sim 33 \times 10^{-17} \mathrm{erg} \mathrm{s}^{-1} \mathrm{~cm}^{-2} \operatorname{arcsec}^{-2}$. The nature of the detected emission and its spatial correlation with the radio emission suggests its identification as emission related to the supernova remnant G 73.9+0.9.

An area of interest where a long-slit spectrum was also obtained is the bright diffuse arc which also overlaps the radio emission (area II in Fig. 2). Counts along a $\sim 1^{\prime}$ aperture were collected to maximize the signal to noise and the area towards the north end of the slit was used for sky background subtraction. Emission from neutral, low and medium ionization species are detected in these spectra (Table 2). The standard diagnostic ratio of $[\mathrm{S} \mathrm{II}] / \mathrm{H} \alpha$ is $\sim 0.5$ suggesting that the diffuce arc-like emission may result from shock heated gas.

Finally, area III (Fig. 5) was chosen due to the presence of a filamentary structure detected in the [O III] image. It was considered that a spectrum would help to identify its nature and clarify its possible relation to G 73.9+0.9. Indeed, the two deep long-slit spectra show that the detected emission results from photoionization processes ( $\left[\mathrm{S} \mathrm{II}_{\mathrm{II}}\right] \mathrm{H} \alpha \sim 0.30$, Table 2 ). In addition, the absence of $\mathrm{H} \beta$ emission $(\mathrm{H} \alpha / \mathrm{H} \beta>8$ at the $3 \sigma$ confidence level) suggests that a distant $\mathrm{H}_{\text {II }}$ filamentary structure was observed and it is not related to the remnant under examination. The H $\alpha$ flux of $9.2 \times 10^{-17} \mathrm{erg} \mathrm{s}^{-1} \mathrm{~cm}^{-2} \mathrm{arcsec}^{-2}$ is the lowest among the $\mathrm{H} \alpha$ fluxes measured in the long-slit spectra obtained.

According to Table 2 the ratio of the sulfur lines approaches the low density limit indicating low electron densities. The spectrum from area I suggests electron densities below $\sim 50 \mathrm{~cm}^{-3}$, while the spectra from areas II and III suggest even lower electron densities. Note here that the signal to noise ratios given in Table 2 do not incorporate errors due to the calibration process which are $\sim 10 \%$.

\section{Discussion}

The first flux calibrated narrow band CCD images of G 73.9+0.9 in the emission lines of $\mathrm{H} \alpha+\left[\mathrm{N}_{\mathrm{II}}\right]$, [ $\left.\mathrm{S}_{\mathrm{II}}\right],\left[\mathrm{O}_{\mathrm{II}}\right]$, and $[\mathrm{O} \mathrm{III}]$ are presented along with deep long-slit spectra. This remnant is located in the Cygnus constellation, a rather complex area of the sky where a large number of dark and bright nebulae are found.

The low ionization images are characterized by diffuse emission, while an $\mathrm{H}_{\text {II }}$ region is present in the west. The [S II] and $\mathrm{H} \alpha+[\mathrm{N} \mathrm{II}]$ images provide the first coarse evidence on the nature of the emission in the central areas of the field observed. They suggest that the arc-like emission originates from shock heated gas as a result of the interaction of the primary blast wave with intestellar "clouds". In this case it is very likely that these "clouds" are seen closer to face on than edge on, an orientation which would result in a filamentary structure (e.g. Hester 1987). In addition, these images allow to identify a small scale structure which emits even stronger in sulfur with respect to $\mathrm{H} \alpha$ than the arc-like structure. This is verified by the deep optical spectra (area I; Table 2) which display the characteristic signature of emission from shock heated gas ([S II $] / \mathrm{H} \alpha \sim 0.7$ ). Given the spatial coincidence of these structures with the radio emission and their spectral signature it is very probable that they are associated to G 73.9+0.9. A new filamentary structure, unknown up to now, has been detected in the medium ionization line of $[\mathrm{O}$ III] to the south of $\mathrm{G} 73.9+0.9$. However, the optical 
Table 2. Relative line fluxes.

\begin{tabular}{|c|c|c|c|c|c|}
\hline & Area I & & Area II & & Area III \\
\hline Line $(\AA)$ & $F^{\mathrm{a}, \mathrm{b}}$ & $I_{\text {corr }}^{\mathrm{c}}$ & $F^{\mathrm{a}, \mathrm{b}}$ & $I_{\text {corr }}^{\mathrm{c}}$ & $F^{\mathrm{a}, \mathrm{b}}$ \\
\hline $4861 \mathrm{H} \beta$ & $18(46)$ & 100 & $22(147)$ & 100 & - \\
\hline $4959[\mathrm{O} \text { III }]_{1}$ & - & - & $1(10)$ & 4.4 & $13(4)$ \\
\hline $5007[\mathrm{O} \text { III }]_{2}$ & - & - & $3.8(38)$ & 16.6 & $67(16)$ \\
\hline $5872 \mathrm{He}$ I & - & - & $2.2(34)$ & 8.0 & - \\
\hline $6300[\mathrm{OI}]_{1}$ & $5(3)$ & 16 & $2.3(28)$ & 7.2 & - \\
\hline $6364[\mathrm{OI}]_{2}$ & - & - & $0.7(13)$ & 2.2 & - \\
\hline $6548\left[\mathrm{~N}_{\mathrm{II}}\right]_{1}$ & $18(77)$ & 54 & $17(214)$ & 51 & $14(7)$ \\
\hline $6563 \mathrm{H} \alpha$ & $100(289)$ & 300 & $100(769)$ & 300 & $100(20)$ \\
\hline $6584\left[\mathrm{~N} \mathrm{II}_{2}\right.$ & $60(231)$ & 179 & $58(611)$ & 173 & $55(14)$ \\
\hline $6678 \mathrm{He} \mathrm{I}$ & - & - & $0.7(14)$ & 2.1 & - \\
\hline $6716\left[\mathrm{~S}_{\mathrm{II}}\right]_{1}$ & $38(103)$ & 109 & $31(349)$ & 90 & $17(8)$ \\
\hline $6731\left[\mathrm{~S} \mathrm{II}_{2}\right.$ & $28(118)$ & 80 & $22(273)$ & 64 & $11(7)$ \\
\hline Absolute $\mathrm{H} \alpha$ flux $^{\mathrm{d}}$ & 33.0 & & 96.2 & & 9.2 \\
\hline $\mathrm{H} \alpha / \mathrm{H} \beta$ & $5.6(45)$ & & $4.55(144)$ & & $>8$ \\
\hline$c$ & $0.81[0.03]$ & & $0.55[0.01]$ & & - \\
\hline$[\mathrm{O}$ III $] / \mathrm{H} \beta$ & - & & $0.22(33)$ & & - \\
\hline$[\mathrm{S}$ II $] / \mathrm{H} \alpha$ & $0.66(133)$ & & $0.53(383)$ & & $0.28(9)$ \\
\hline$I(6716) / I(6731)$ & $1.36(77)$ & & $1.40(215)$ & & $1.55(5)$ \\
\hline
\end{tabular}

${ }^{\text {a }}$ Fluxes uncorrected for interstellar extinction and relative to $F(\mathrm{H} \alpha)=100$.

${ }^{\mathrm{b}}$ Listed fluxes are a signal to noise weighted average of the individual fluxes.

${ }^{\mathrm{c}}$ Corrected for interstellar extinction.

${ }^{\mathrm{d}}$ In units of $10^{-17} \mathrm{erg} \mathrm{s}^{-1} \mathrm{~cm}^{-2} \operatorname{arcsec}^{-2}$.

Numbers in parentheses represent the signal to noise ratio of the quoted fluxes.

The $1 \sigma$ error is given in square brackets for the logarithmic extinction.

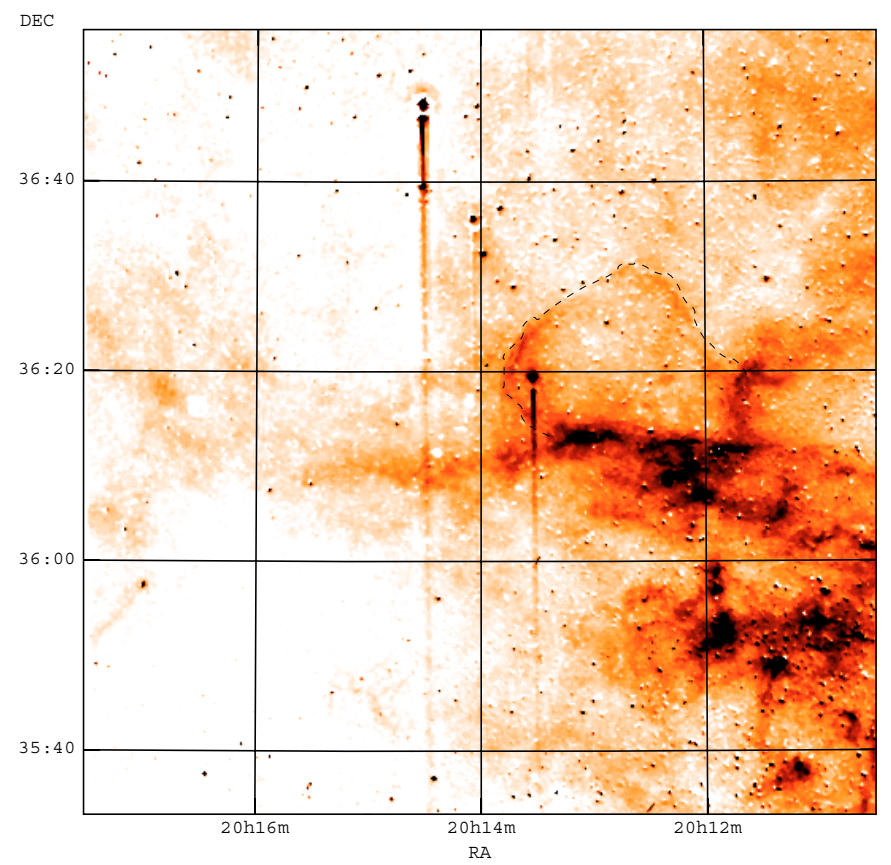

Fig. 3. The morphology in the emission lines of [O II] 3726 and $3729 \AA$ is also diffuse, similar to the low ionization images. The image has been smoothed to suppress the residuals from the imperfect continuum subtraction and the shadings run linearly from 2 to $50 \times 10^{-17} \mathrm{erg} \mathrm{s}^{-1} \mathrm{~cm}^{-2} \operatorname{arcsec}^{-2}$. The dash line outlines the faint broken loop of emission discussed in Sect. 3.2.

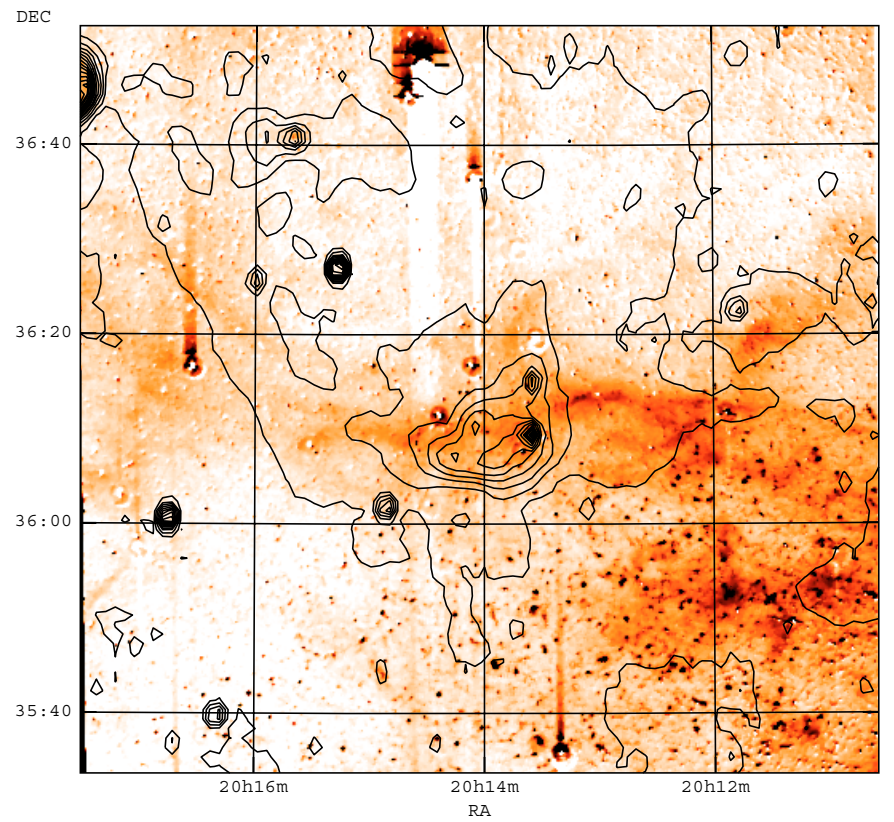

Fig. 4. The correlation between the sulfur line emission and the radio emission at $1420 \mathrm{MHz}$ is shown in this figure. No significant optical emission is detected to the north where radio emission is also present and was initially proposed to be part of G 73.9+0.9. The $1420 \mathrm{MHz}$ contours display the brightness temperature and run from 4 to $30 \mathrm{~K}$. The resolution of the radio data is $1.0 \times 1.7 \operatorname{arcmin}^{2}$ (Pineault \& Chastenay 1990). 


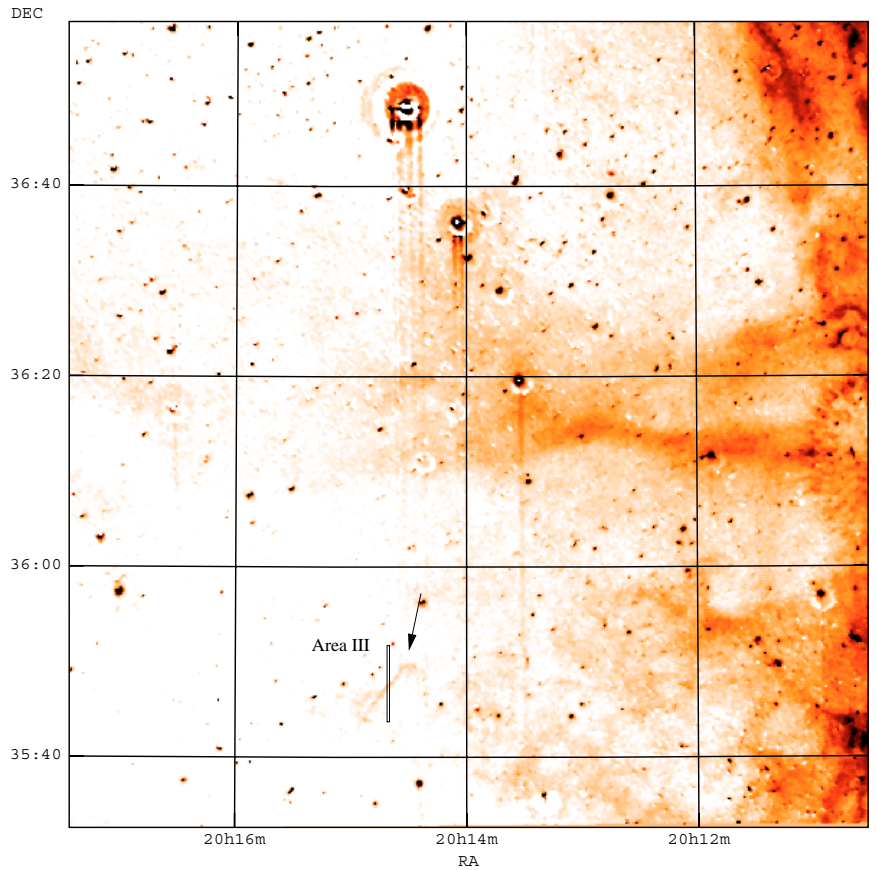

Fig. 5. The medium ionization line of [O $\mathrm{O}_{\text {III }} 5007 \AA$ reveals significant emission along the west edge of the observed field. The image has been smoothed to suppress the residuals from the imperfect continuum subtraction and the shadings run linearly from 0 to $35 \times 10^{-17} \mathrm{erg} \mathrm{s}^{-1} \mathrm{~cm}^{-2} \operatorname{arcsec}^{-2}$. The arrow points to the filamentary structure discovered during these imaging observations, while the long rectangle marks the approximate projection of the slit on the sky.

spectrum of this filament suggests that it is a background $\mathrm{H}_{\text {II }}$ structure without any relation to this remnant (area III in Fig. 5; Table 2). It is also situated well outside the faintest radio contours of the remnant.

The spectra from areas I and II indicate that the observed shock structures are complete since the $[\mathrm{O} \mathrm{III}] / \mathrm{H} \beta$ ratio does not exceed a value of $\sim 6$ (e.g. Raymond et al. 1988). Shock structures with higher $[\mathrm{O}$ III] $/ \mathrm{H} \beta$ ratios than this value are representative of incomplete shock structures. The ratio of the sulfur lines at 6716 and $6731 \AA$ allows to estimate the electron density in a low ionization region. Both ratios are close to the upper end of the allowable range of values suggesting very low electron densities $\left(<50 \mathrm{~cm}^{-3}\right)$. The low electron densities, the weak to absent [O III] emission and the shock modeling of Hartigan et al. (1987) and Raymond et al. (1988) imply shock velocities below $90 \mathrm{~km} \mathrm{~s}^{-1}$, probably around $70-80 \mathrm{~km} \mathrm{~s}^{-1}$, and low preshock cloud densities of the order of a few atoms per $\mathrm{cm}^{3}$. Finally, the above models also suggest that moderate magnetic fields $(\sim 15-20 \mu \mathrm{G})$ should be present to match the observational data. The effect of the magnetic field in a shock structure is to limit the compression and stretch the recombination zone (e.g. Cox 1972). Limited compression implies lower densities behind the shock front in agreement with the low electron densities implied by the large sulfur line ratios.

The $\mathrm{H} \alpha / \mathrm{H} \beta$ ratios of 5.6 and 4.6 towards the observed areas are indicative of the amount of interstellar extinction that the optical radiation suffers. These ratios do not differ significantly, if the calibration errors $(\sim 10 \%)$ of the long-slit spectra are taken into account, and are equivalent to a reddening $E(B-V)$ of 0.54 and 0.37 , respectively. Statistical studies of the optical reddening and the $\mathrm{X}$-ray determined hydrogen column density show a linear relation between these quantities (e.g. Ryter et al. 1975; Predehl \& Schmitt 1995). The hydrogen column density $N_{\mathrm{H}}$ can be estimated through the relation of

$N_{\mathrm{H}}=5.4( \pm 0.1) \times 10^{21} E(B-V) \mathrm{cm}^{-2}$,

given by Predehl and Schmitt (1995) and substituting $E(B-V)$ with the measured values. The column densities that result from this formula are 2.9 and $2.0 \times 10^{21} \mathrm{~cm}^{-2}$. The total galactic column density is $\sim 1.2 \times 10^{22} \mathrm{~cm}^{-2}$ according to the web tool in HEASARC (http://legacy.gsfc.nasa.gov) which is based on the work of Dickey \& Lockmann (1990). A similar web tool which calculates the column density out to a certain distance is available from the Space Telescope Science Institute (http: //archive.stsci.edu) and is based on the work of Fruscione et al. (1994) and Diplas \& Savage (1994). A column density of $\sim 2 \times 10^{21} \mathrm{~cm}^{-2}$ is calculated for a distance of $2 \mathrm{kpc}$ using this tool. Our measurements are compatible with the total galactic column density and are in fairly good agreement with the column density accumulated up to a distance of $2 \mathrm{kpc}$, which is also compatible with the range of distances estimated by Lozinskaya et al. (1993) based on kinematic measurements. Assuming a distance of $2 \mathrm{kpc}$, an average ISM density of $\sim 0.5 \mathrm{~cm}^{-3}$ would be implied along the line of sight. Clearly, it is difficult to estimate the distance given the complexity of the Cygnus area and the statistical nature of the equations involved. However, the estimated column densities are probably of the correct order.

The area of G 73.9+0.9 was observed by ROSAT in the course of the All-Sky survey for $\sim 720$ s. No soft X-ray emission is especially prominent in the field and an overlay with the radio contours does not provide any additional information. A rough $3 \sigma$ upper limit on the soft $\mathrm{X}$-ray emission from this remnant in the ROSAT band is $\sim 1.5 \times 10^{-3} \mathrm{cts} \mathrm{s}^{-1} \operatorname{arcmin}^{-2}$. The failure to detect $\mathrm{X}$-ray emission prevents the determination of the local ISM density and explosion energy through the Sedov-Taylor solution even if the distance were accurately known. The absence of soft X-ray emission may indicate a low shock temperature and/or a low density of the local interstellar medium. In this case the average ISM density of $\sim 0.5 \mathrm{~cm}^{-3}$ estimated earlier would seem reasonable and would imply a density contrast of $\sim 10$ between the ISM and the interstellar "clouds". If, in addition, the condition for pressure equilibrium holds then the primary shock velocity would lie in the range of $200-300 \mathrm{~km} \mathrm{~s}^{-1}$.

\section{Conclusions}

Diffuse emission is the major characteristic of the field of the supernova remnant G 73.9+0.9. The low ionization images further indicate an inhomogenous and patchy intestellar medium. A new filamentary structure is detected in the [O III] image but is not related to the remnant. Long-slit spectra suggest complete recombination zones, shock velocities below $90 \mathrm{~km} \mathrm{~s}^{-1}$, and low electron densities. They also allow to identify the bright arc and a small scale structure in the east part of the 
remnant as emission related to $\mathrm{G} 73.9+0.9$ on the basis of their positional coincidence and spectral nature. The optical extinction suggests an equivalent hydrogen column density of a few times $10^{21} \mathrm{~cm}^{-2}$.

Acknowledgements. We would like to thank the referee Serge Pineault for his comments and for providing the $1420 \mathrm{MHz}$ digital data. Skinakas Observatory is a collaborative project of the University of Crete, the Foundation for Research and Technology-Hellas and the Max-Planck-Institut für Extraterrestrische Physik. This research has made use of data obtained through the High Energy Astrophysics Science Archive Research Center Online Service, provided by the NASA/Goddard Space Flight Center.

\section{References}

Aller, L. H. 1984, Physics of thermal gaseous nebulae (D. Reidel Publishing Company)

Boumis, P., Mavromatakis, F., \& Paleologou, E. V. 2002, A\&A, 385, 1042

Cox, D. P., \& Raymond, J. C. 1985, ApJ, 298, 651

Cox, D. P. 1972, ApJ, 178, 143

Dickey, J. M., \& Lockman, F. J. 1990, ARA\&A, 28, 215

Diplas, A., \& Savage, B. D. 1994, ApJS, 93, 211

Fruscione, A., Hawkins, I., Jelinsky, P., \& Wiercigroch, A. 1994, ApJS, 94, 127
Gervais, S., \& St-Louis, N. 1999, AJ, 118, 2394

Hartigan, P., Raymond, J., \& Hartmann, L. 1987, ApJ, 316, 323

Hamuy, M., Walker, A. R., Suntzeff, N. B., et al. 1992, PASP, 104, 533

Hamuy, M., Suntzeff, N. B., Heathcote, S. R., et al. 1994, PASP, 106, 566

Hester, J. 1987, ApJ, 314, 187

Hunter, \& Deidre, A. 1992, ApJS, 79, 469

Kaler, J. B. 1976, ApJS, 31, 517

Lasker, B. M., Russel, J. N., \& Jenkner, H. 1999, in the HST Guide Star Catalog, version 1.1-ACT, The Association of Universities for Research in Astronomy, Inc.

Lozinskaya, T. A., Sitnik, T. G., \& Pravdikova, V. V. 1993, Astron. Rep., 37, 3

Mavromatakis, F., Boumis, P., \& Paleologou, E. V. 2002, A\&A, 385, 1042

Pineault, S. 1998, AJ, 115, 2483

Pineault, S., \& Chastenay, P. 1990, MNRAS, 246, 169

Pineault, S., Gaumont-Guay, S., \& Madore, B. 1996, AJ, 112, 201

Predehl, P., \& Schmitt, J. H. M. M. 1995, A\&A, 293, 889

Raymond, J. C., Hester, J. J., Cox, D., et al. 1988, ApJ, 324, 869

Reich, W., Fur̈st, E., Reich, P., Sofue, Y., \& Handa, T. 1986, A\&A, 155,185

Ryter, C., Cesarsky, C. J., \& Audouze, J. 1975, ApJ, 198, 103

Smith, R. C., Kirshner, R. P., Blair, W. P. Long, K. S., \& Winkler, P. F. 1993, ApJ, 407, 564 